\title{
Some Fixed Point Results on b-(E.A.) Property of Integral Type Mappings in b-Metric Spaces
}

\section{Amarjeet Singh Saluja}

Institute for Excellence in Higher Education, Bhopal, Madhya Pradesh, India.

\begin{tabular}{ll}
\hline ARTICLE INFO & ABSTRACT \\
\hline Published Online: & In this paper, we confine over selves to obtain some results on fixed point theorems for Lebesgue \\
06 October 2020 & integrable mapping satisfying b-(E.A.) property in b- metric spaces. We have given an example to \\
Corresponding Author: & support our results and also prove that the fixed point problem is well posed for the mappings. \\
A. S. Saluja &
\end{tabular}

KEYWORDS: Fixed Point, b- (E.A.)-Property, Weakly Compatible Mappings, b- Metric Space etc.

Mathematical Subject Classification (2000): Primary 47H10, Secondary 54H25.

\section{Introduction}

During the last 95- years a lot of fixed point results have been established and we find that Banach contraction principle is at the base of the most of these results established so far. The concept of metric spaces has been generalized in many directions. The notion of a b-metric space was introduced by Czerwik in [12,13] and during the last few years by many authors a lot of fixed point theorems have been proved by many authors in b-metric spaces. In the year 1986, Jungck [18] introduced the notion of compatible mappings and utilized the same to improve commutativity conditions in common fixed point theorems. This concept has been frequently employed to prove existence theorems on common fixed points. However, the study of common fixed points of non-compatible mappings was initiated by Pant [24]. Recently, Aamri and Moutawakil [1] and Liu et al. [29] respectively defined the property (E.A) and the common property (E.A) and established fruitful results on common fixed point theorems in metric spaces. Later some authors introduced some new fixed point results using this concept like, Ali et al. [5], Babu and Sailaja [7], Nazir and Abbas [21], Oztirk and Radanovic [22], Ozturk and Turkoglu [23]. The following definitions are required in sequel.

\section{Preliminaries}

Definition 2.1 [8] Let $X$ be a nonempty set. A mapping $d$ : $\mathrm{X} \times \mathrm{X} \rightarrow[0, \infty)$ is called $\mathrm{b}$-metric if there exists a real number $\mathrm{b} \geq 1$ such that for every $\mathrm{x}, \mathrm{y}, \mathrm{z} \in \mathrm{X}$, we have

(i) $\quad d(x, y)=0$ if and only if $x=y$

(ii) $\mathrm{d}(\mathrm{x}, \mathrm{y})=\mathrm{d}(\mathrm{y}, \mathrm{x})$ (iii) $\quad d(x, z) \leq b[d(x, y)+d(y, z)]$

In this case the pair $(X, d)$ is called a $b$-metric space. There exists many examples in the literature (see[6-8])(BS) showing that every metric function is a $b$-metric function with $b=1$, while the converse is not true, i.e. the class of $b$ - metric functions is effectively larger than that of ordinary metric function.

Definition 2.2 [17] Let $\left\{x_{n}\right\}$ be a sequence in a b-metric space $(X, d)$.

(i) $\quad\left\{\boldsymbol{x}_{\boldsymbol{n}}\right\}$ is called b-convergent if and only if there is $\mathrm{X} \in$ $\mathrm{X}$ such that $\mathrm{d}\left(\boldsymbol{x}_{\boldsymbol{n}}, \boldsymbol{x}\right) \rightarrow 0$ as $\mathrm{n} \rightarrow \infty$.

(ii) $\quad\left\{\boldsymbol{x}_{\boldsymbol{n}}\right\}$ is called b-Cauchy sequence if and only if $\mathrm{d}\left(\boldsymbol{x}_{\boldsymbol{n}}, \boldsymbol{x}_{\boldsymbol{m}}\right) \rightarrow 0$ as $\mathrm{n}, \mathrm{m} \rightarrow \infty$.

A b-metric space $(\mathrm{X}, \mathrm{d})$ is said to be complete if and only if each b-Cauchy sequence in $\mathrm{X}$ is b-convergent.

Definition 2.3 [16] Let $(X, d)$ be a $b$-metric space. A subset $\mathrm{Y} \subset \mathrm{X}$ is called closed if and only if for each sequence $\left\{\mathrm{xn}_{\mathrm{n}}\right\}$ in $Y$ which $b$-converges to an element $x$, we have $\mathrm{x} \in \mathrm{Y}$.

Definition 2.4 [18] Let $(X, d)$ be a b-metric space and $f$ and $g$ are self-maps on $\mathrm{X}$.

(i) $\mathrm{f}$ and $\mathrm{g}$ are said to be compatible if whenever a sequence $\left\{x_{n}\right\}$ in $X$ is such that $\left\{\mathrm{fx}_{\mathrm{n}}\right\}$ and $\left\{\mathrm{gx}_{\mathrm{n}}\right\}$ are b- convergent to some $t \in X$, then $\lim _{n \rightarrow \infty} d\left(f g x_{n}, g f x_{n}\right)=0$.

(ii) $\mathrm{f}$ and $\mathrm{g}$ are said to non-compatible if there exists at least one sequence $\left\{x_{n}\right\}$ in $X$ is such that $f x_{n}$ and $\mathrm{gx}_{\mathrm{n}}$ are $\mathrm{b}$-convergent to some $\mathrm{t} \in \mathrm{X}$, but 
"Some Fixed Point Results on b-(E.A.) Property of Integral Type Mappings in b-Metric Spaces"

$\lim _{n \rightarrow \infty} d\left(f g x_{n}, g f x_{n}\right)$ is either non zero or does not exist.

Definition 2.5 [23] $f$ and $g$ are said to satisfy the b-(E.A) property if there exists a sequence $\left\{\mathrm{x}_{\mathrm{n}}\right\}$ such that $\lim _{n \rightarrow \infty} \operatorname{fgx}_{n}=\lim _{n \rightarrow \infty} g x_{n}=t$, for some $t \in X$.

Remark 2.6 Non-compatibility implies b-(E.A)-property. Example 2.7 [23] Let $\mathrm{X}=[0,2]$ and define $\mathrm{d}: \mathrm{X} \times \mathrm{X} \rightarrow$ $[0, \infty)$ as follows

$$
d(x, y)=(x-y)^{2}
$$

Let $\mathrm{f}, \mathrm{g}: \mathrm{X} \rightarrow \mathrm{X}$ be defined by

$$
\mathrm{f}(\mathrm{x})=\left\{\begin{array}{ll}
1, & x \in[0,1] \\
\frac{x+1}{8}, & x \in(1,2]
\end{array} \text { and } \mathrm{g}(\mathrm{x})= \begin{cases}\frac{3-x}{2}, & x \in[0,1] \\
\frac{x}{4}, & x \in(1,2]\end{cases}\right.
$$

for a sequence $\left\{x_{n}\right\}$ in $\mathrm{X}$ such that $x_{n}=1+\frac{1}{n_{+} 2}, \mathrm{n}=0,1$, $2, .$.

and $\lim _{n \rightarrow \infty} \mathrm{f} x_{n}=\lim _{n \rightarrow \infty} \mathrm{g} x_{n}=\frac{1}{4}$

So $f$ and $g$ are satisfying the $b$-(E.A) property.

$\lim _{n \rightarrow \infty} \mathrm{d}\left(\mathrm{fg} x_{n}, \mathrm{gf} x_{n}\right)$ exists and it is not equal to 0 . Thus $f$ and $g$ are non-compatible.

Definition 2.8 [2] Let $\mathrm{f}$ and $\mathrm{g}$ be self-maps of a set $\mathrm{X}$. If $\mathrm{w}=$ $\mathrm{f} x=\mathrm{gx}$ for some $\mathrm{x}$ in $\mathrm{X}$ then $\mathrm{x}$ is called a coincidence point of $f$ and $g$ and $w$ is called a point of coincidence of $f$ and $g$.

Definition 2.9 [2] Let $\mathrm{f}$ and $\mathrm{g}$ be self-maps of a set $\mathrm{X}$. Then $\mathrm{f}$ and $g$ are said to be weakly compatible if they commute at their coincidence point.

Proposition 2.10 [2] Let $\mathrm{f}$ and $\mathrm{g}$ be weakly compatible selfmaps of a set $X$. If $f$ and $g$ have a unique point of coincidence $\mathrm{w}=\mathrm{f} \mathrm{x}=\mathrm{gx}$, then $\mathrm{w}$ is the unique common fixed point of $\mathrm{f}$ and $\mathrm{g}$.

Definition 2.11. [5] A function $\phi$ is said to be integral sub additive, if for each $\alpha, \beta>0$,

$$
\int_{0}^{\alpha+\beta} \varphi(\mathrm{t}) \mathrm{dt} \leq \int_{0}^{\alpha} \varphi(\mathrm{t}) \mathrm{dt}+\int_{0}^{\beta} \varphi(\mathrm{t}) \mathrm{dt}
$$

Lemma 2.12. [28] Let $\phi:[0, \infty) \rightarrow[0, \infty]$ be Lebesgue integrable mapping which is summable on each compact subset of $[0, \infty)$, non-negative and such that for each $\varepsilon>0$, $\int_{0}^{c} \varphi(t) d t>0$ and $\left\{a_{n}\right\}$ be a sequence of non-negative numbers with $\lim _{n \rightarrow \infty} a_{n}=\mathrm{u}$. Then

$$
\lim _{n \rightarrow \infty} \int_{0}^{a_{n}} \varphi(\mathrm{t}) \mathrm{dt}=\int_{0}^{\mathrm{a}} \varphi(\mathrm{t}) \mathrm{dt}
$$

Lemma 2.13. [28] Let $\phi:[0, \infty) \rightarrow[0, \infty]$ be Lebesgue integrable mapping which is summable on each compact subset of $[0, \infty)$, non-negative and such that for each $\varepsilon>0$, $\int_{0}^{c} \varphi(t) d t>0$ and $\left\{a_{n}\right\}$ be a sequence of non-negative numbers with $\lim _{n \rightarrow \infty} a_{n}=\mathrm{u}$. Then $\lim _{n \rightarrow \infty} \int_{0}^{a_{n}} \varphi(\mathrm{t}) \mathrm{dt} \Leftrightarrow$ $\lim _{n \rightarrow \infty} a_{n}=0$

We define $\psi$ and $\Phi$ as follows: $\psi=\{\psi:[0, \infty) \rightarrow[0, \infty): \psi$ is upper semi-continuous, sequence $\psi^{\mathrm{n}}(\mathrm{t})$ converges to 0 as $\mathrm{n} \rightarrow \infty, \forall \mathrm{t}>0$ and $\psi(\mathrm{t})$ $<\mathrm{t}$ for any $\mathrm{t}>0\}$
$\Phi=\{\varphi:[0, \infty) \rightarrow[0, \infty]: \phi$ is Lebesgue integrable, summable on each compact subset of $[0, \infty)$, non-negative and for each $\left.\varepsilon>0, \int_{0}^{c} \varphi(t) d t>0\right\}$

throughout in this paper we shall making use of the standard notations and terminologies of nonlinear analysis.

\section{Main results}

Theorem 3.1 Let $(\mathrm{x}, \mathrm{d})$ be $\mathrm{b}$ - metric space with $\mathrm{b}>1$ and $\mathrm{f}$, $\mathrm{g}, \mathrm{S}, \mathrm{T}: \mathrm{X} \rightarrow \mathrm{X}$ be mappings with $f(X) \subseteq T(X)$ and $g(X) \subseteq S(X)$ such that

$$
\int_{0}^{b^{\varepsilon} d(f x, g y)} \emptyset(t) d t \leq \int_{0}^{M_{b}(x, y)} \emptyset(t) d t \quad \forall \mathrm{x}, \mathrm{y} \in
$$

Where $\varepsilon>1$ is a constant and $\emptyset:[0, \infty) \rightarrow[0, \infty)$ is Lebesque integrable mapping which is summable on each compact subset of $[0, \infty)$, non-negative and such that for $\mathrm{c}>0$, $\int_{0}^{c} \emptyset(t) d t>0$ and

$$
\begin{gathered}
\text { (3.1.2) } M_{b}(x, y)=\max \{\mathrm{d}(\mathrm{Sx}, \mathrm{Ty}), \mathrm{d}(\mathrm{fx}, \mathrm{Sx}), \\
\left.\mathrm{d}(\mathrm{gy}, \mathrm{Ty}), \frac{\mathrm{d}(\mathrm{gy}, \mathrm{Ty})+\mathrm{d}(\mathrm{fx}, \mathrm{Sx})}{2 b}, \frac{\mathrm{d}(\mathrm{Sx}, \mathrm{gy})+\mathrm{d}(\mathrm{fx}, \mathrm{Ty})}{2 b}\right\}
\end{gathered}
$$

Suppose that one of the pair $(f, S)$ and $(g, T)$ satisfy the b(E.A.)-property and that one of the subspaces $f(X), g(X)$, $T(X)$ and $S(X)$ is b-closed in $X$. Then the pair $(f, S)$ and $(g, T)$ have a point of Coincidence in X, Moreover, if the pair $(f, S)$ and $(g, T)$ are weakly compatible, then $\mathrm{f}, \mathrm{g}, \mathrm{S}$ and $\mathrm{T}$ have a unique common fixed point.

Proof : If the pair $(f, S)$ satisfies the b-(E.A.)-property, then there exists a sequence $\left\{x_{n}\right\}$ in $\mathrm{X}$ satisfying

$$
\lim _{n \rightarrow \infty} f x_{n}=\lim _{n \rightarrow \infty} S x_{n}=\mathrm{q} \text {, for some } \mathrm{q} \in \mathrm{X} \text {. }
$$

As $f(X) \subseteq T(X)$ there exists a sequence $\left\{y_{n}\right\}$ in $X$ such that $f x_{n}=T y_{n}$

$$
\text { Hence, } \lim _{n \rightarrow \infty} T y_{n}=\mathrm{q} \text {. }
$$

Let us show that $\lim _{n \rightarrow \infty} g y_{n}=\mathrm{q}$.

By (3.1.1),

$$
\begin{gathered}
\int_{0}^{b^{\varepsilon} d\left(f x_{n}, g y_{n}\right)} \emptyset(t) d t \leq \int_{0}^{M_{b}\left(x_{n}, y_{n}\right)} \emptyset(t) d t \\
\text { Where, } \\
M_{b}\left(x_{n}, y_{n}\right)=\max \\
\left\{\mathrm{d}\left(\mathrm{S} x_{n}, \mathrm{~T} y_{n}\right), \mathrm{d}\left(\mathrm{f} x_{n}, \mathrm{~S} x_{n}\right), \mathrm{d}\left(\mathrm{g} y_{n}, \mathrm{~T} y_{n}\right), \frac{\mathrm{d}\left(\mathrm{g} y_{n}, \mathrm{~T} y_{n}\right)+\mathrm{d}\left(\mathrm{f} x_{n}, \mathrm{~S} x_{n}\right)}{2 b},\right. \\
\left.\frac{\mathrm{d}\left(\mathrm{S} x_{n}, \mathrm{~g} y_{n}\right)+\mathrm{d}\left(\mathrm{f} x_{n}, \mathrm{~T} y_{n}\right)}{2 b}\right\} \\
=\max \left\{\mathrm{d}\left(\mathrm{S} x_{n}, f x_{n}\right), \mathrm{d}\left(\mathrm{f} x_{n}, \mathrm{~S} x_{n}\right), \mathrm{d}\left(\mathrm{g} y_{n},\right.\right. \\
\left.\left.f x_{n}\right), \frac{\mathrm{d}\left(\mathrm{g} y_{n}, f x_{n}\right)+\mathrm{d}\left(\mathrm{f} x_{n}, \mathrm{~S} x_{n}\right)}{2 b}, \frac{\mathrm{d}\left(\mathrm{S} x_{n}, \mathrm{~g} y_{n}\right)+\mathrm{d}\left(\mathrm{f} x_{n}, f x_{n}\right)}{2 b}\right\} \\
\leq \max \left\{\mathrm{d}\left(\mathrm{f} x_{n}, \mathrm{~S} x_{n}\right), \mathrm{d}\left(\mathrm{g} y_{n}, f x_{n}\right), \frac{\mathrm{d}\left(\mathrm{g} y_{n}, f x_{n}\right)+\mathrm{d}\left(\mathrm{f} x_{n}, \mathrm{~S} x_{n}\right)}{2 b},\right. \\
\left.\frac{\mathrm{b}\left[\mathrm{d}\left(\mathrm{S} x_{n}, \mathrm{f} x_{n}\right)+\mathrm{d}\left(\mathrm{f} x_{n}, \mathrm{~g} y_{n}\right)\right]}{2 b}\right\} \\
=\max \left\{\mathrm{d}\left(\mathrm{f} x_{n}, \mathrm{~S} x_{n}\right), \mathrm{d}\left(\mathrm{g} y_{n}, f x_{n}\right)\right\}
\end{gathered}
$$

In (3.1.3), on taking limit and using lemma 2.13, we obtain

$$
\begin{aligned}
& \lim _{n \rightarrow \infty} \int_{0}^{b^{\varepsilon} d\left(f x_{n}, g y_{n}\right)} \emptyset(t) d t \\
& \quad \leq \lim _{n \rightarrow \infty} \int_{0}^{\max \left\{\mathrm{d}\left(\mathrm{f} x_{n}, \mathrm{~S} x_{n}\right), \mathrm{d}\left(\mathrm{g} y_{n}, f x_{n}\right)\right\}} \emptyset(t) d t
\end{aligned}
$$


"Some Fixed Point Results on b-(E.A.) Property of Integral Type Mappings in b-Metric Spaces"

$=\lim _{n \rightarrow \infty} \int_{0}^{\mathrm{d}\left(\mathrm{g} y_{n}, f x_{n}\right)} \emptyset(t) d t, \quad$ because $\mathrm{d}\left(\mathrm{f} x_{n}, \mathrm{~S} x_{n}\right) \rightarrow 0$ as $\mathrm{n}$ $\rightarrow \infty$.

Since $b^{\varepsilon}>\mathrm{b}>1$, we have therefore

$\lim _{n \rightarrow \infty} g y_{n}=\lim _{n \rightarrow \infty} f x_{n}=\mathrm{q}$

Further, we have

$$
\frac{1}{b} d\left(q, g y_{n}\right) \leq d\left(q, f x_{n}\right)+d\left(f x_{n}, g y_{n}\right) \rightarrow 0 \text { as } \mathrm{n} \rightarrow \infty .
$$

Thus, $g y_{n} \rightarrow \mathrm{q}$ as $\mathrm{n} \rightarrow \infty$.

If $\mathrm{T}(\mathrm{X})$ is closed subspace of $\mathrm{x}$, then there exists a $r \in X$, such that $\operatorname{Tr}=\mathrm{q}$.

Now, we shall show that $\mathrm{gr}=\mathrm{q}$. Indeed, we have

$$
\frac{1}{b} d(q, g r) \leq d\left(q, f x_{n}\right)+d\left(f x_{n}, g r\right),
$$

Or,

(3.1.4)

$$
\frac{1}{b} d(q, g r) \leq d\left(q, f x_{n}\right)+\frac{1}{b^{\varepsilon}} M_{b}\left(x_{n}, r\right)
$$

Where,

$$
\begin{gathered}
M_{b}\left(x_{n}, r\right)=\max \\
\left\{\mathrm{d}\left(\mathrm{S} x_{n}, \mathrm{~T} r\right), \mathrm{d}\left(\mathrm{f} x_{n}, \mathrm{~S} x_{n}\right), \mathrm{d}(\mathrm{g} r, \mathrm{~T} r), \frac{\mathrm{d}(\mathrm{g} r, \mathrm{~T} r)+\mathrm{d}\left(\mathrm{f} x_{n}, \mathrm{~S} x_{n}\right)}{2 b},\right. \\
\left.\frac{\mathrm{d}\left(\mathrm{S} x_{n}, \mathrm{~g} r\right)+\mathrm{d}\left(\mathrm{f} x_{n}, \mathrm{~T} r\right)}{2 b}\right\} \\
=\max \left\{\mathrm{d}\left(\mathrm{S} x_{n}, \mathrm{q}\right), \mathrm{d}\left(\mathrm{f} x_{n}, \mathrm{~S} x_{n}\right), \mathrm{d}(\mathrm{g} r, \mathrm{q}), \frac{\mathrm{d}(\mathrm{g} r, \mathrm{q})+\mathrm{d}\left(\mathrm{f} x_{n}, \mathrm{~S} x_{n}\right)}{2 b},\right. \\
\left.\frac{\mathrm{d}\left(\mathrm{S} x_{n}, \mathrm{~g} r\right)+\mathrm{d}\left(\mathrm{f} x_{n}, \mathrm{q}\right)}{2 b}\right\} \\
\leq \max \left\{\mathrm{d}\left(\mathrm{S} x_{n}, \mathrm{q}\right), \mathrm{d}\left(\mathrm{f} x_{n}, \mathrm{~S} x_{n}\right), \mathrm{d}(\mathrm{g} r, \mathrm{q}), \frac{\mathrm{d}(\mathrm{g} r, \mathrm{q})+\mathrm{d}\left(\mathrm{f} x_{n}, \mathrm{~S} x_{n}\right)}{2 b},\right. \\
\left.\frac{\mathrm{b}\left[\mathrm{d}\left(\mathrm{S} x_{n}, \mathrm{q}\right)+\mathrm{d}(\mathrm{q}, \mathrm{gr})\right]+\mathrm{d}\left(\mathrm{f} x_{n}, \mathrm{q}\right)}{2 b}\right\}
\end{gathered}
$$

on taking limit, we obtain

$$
\begin{gathered}
\lim _{n \rightarrow \infty} M_{b}\left(x_{n}, r\right) \leq \max \\
\left\{\mathrm{d}(\mathrm{q}, \mathrm{q}), \mathrm{d}(\mathrm{q}, \mathrm{q}), \mathrm{d}(\mathrm{g} r, \mathrm{q}), \frac{\mathrm{d}(\mathrm{g} r, \mathrm{q})+\mathrm{d}(\mathrm{q}, \mathrm{q})}{2 b},\right. \\
\left.\frac{\mathrm{b}[\mathrm{d}(\mathrm{q}, \mathrm{q})+\mathrm{d}(\mathrm{q}, \mathrm{gr})]+\mathrm{d}(\mathrm{q}, \mathrm{q})}{2 b}\right\} \\
=\mathrm{d}(\mathrm{g} r, \mathrm{q})
\end{gathered}
$$

Then, (3.1.4) implies that

$\frac{1}{b} d(q, g r) \leq 0+\frac{1}{b^{\varepsilon}} \mathrm{d}(\mathrm{g} r, \mathrm{q})$

From which it follows that $\mathrm{d}(\mathrm{g} r, \mathrm{q})=0$ (because $b^{\varepsilon}>\mathrm{b}>1$ ).

Hence, $\mathrm{q}=\mathrm{gr}=\mathrm{Tr}$, i.e. $\mathrm{r}$ is the coincidence point of the pair $(\mathrm{g}, \mathrm{T})$.

As $\mathrm{g}(\mathrm{X}) \subseteq \mathrm{S}(\mathrm{X})$, there exists a point $\mathrm{z} \in \mathrm{X}$ such that $\mathrm{q}=\mathrm{Sz}$.

We claim that $\mathrm{Sz}=\mathrm{fz}$. By (3.1.1), we have

(3.1.5)

$$
\int_{0}^{b^{\varepsilon} d(f z, g r)} \emptyset(t) d t \leq \int_{0}^{M_{b}(z, r)} \emptyset(t) d t
$$

Where,

$$
\begin{gathered}
M_{b}(z, r)=\max \\
\left\{\mathrm{d}(\mathrm{S} z, \mathrm{~T} r), \mathrm{d}(\mathrm{f} z, \mathrm{~S} z), \mathrm{d}(\mathrm{g} r, \mathrm{~T} r), \frac{\mathrm{d}(\mathrm{g} r, \mathrm{~T} r)+\mathrm{d}(\mathrm{f} z, \mathrm{~S} z)}{2 b},\right. \\
\left.\frac{\mathrm{d}(\mathrm{S} z, \mathrm{~g} r)+\mathrm{d}(\mathrm{f} z, \mathrm{~T} r)}{2 b}\right\} \\
=\max \left\{\mathrm{d}(\mathrm{q}, \mathrm{q}), \mathrm{d}(\mathrm{fz}, \mathrm{q}), \mathrm{d}(\mathrm{q}, \mathrm{q}), \frac{\mathrm{d}(\mathrm{q}, \mathrm{q})+\mathrm{d}(\mathrm{f} z, \mathrm{q})}{2 b},\right. \\
\left.\frac{\mathrm{d}(\mathrm{q}, \mathrm{q})+\mathrm{d}(\mathrm{f} z, \mathrm{q})}{2 b}\right\} \\
=\mathrm{d}(\mathrm{f} z, \mathrm{q}) .
\end{gathered}
$$

Therefore from (3.1.5), we have

Or,

$$
\int_{0}^{b^{\varepsilon} d(f z, g r)} \emptyset(t) d t \leq \int_{0}^{\mathrm{d}(\mathrm{fz}, \mathrm{q}) \cdot} \emptyset(t) d t
$$

$$
\int_{0}^{b^{\varepsilon} d(f z, q)} \emptyset(t) d t \leq \int_{0}^{\mathrm{d}(\mathrm{fz}, \mathrm{q}) \cdot} \emptyset(t) d t
$$

From which it follows that $\mathrm{d}(\mathrm{fz}, \mathrm{q})=0$ (because $\left.b^{\varepsilon}>\mathrm{b}>1\right)$.

Therefore, $\mathrm{Sz}=\mathrm{fz}=\mathrm{q}$.

Hence, $z$ is a coincidence point of the pair (f, $S$ ).

Thus, $\mathrm{fz}=\mathrm{Sz}=\mathrm{gr}=\mathrm{Tr}=\mathrm{q}$.

By the weak compatibility of the pair (f, S) and $(g, T)$, we obtain that $\mathrm{fq}=\mathrm{Sq}$ and $\mathrm{gq}=\mathrm{Tq}$.

Now, we shall show that $q$ is a common fixed point off, $g, S$ and $\mathrm{T}$.

From (3.1.1), we have

$$
\begin{gathered}
\int_{0}^{b^{\varepsilon} d(f q, q)} \emptyset(t) d t=\int_{0}^{b^{\varepsilon} d(f q, g r)} \emptyset(t) d t \leq \\
\int_{0}^{M_{S}(q, r)} \emptyset(t) d t
\end{gathered}
$$

Where,

$$
\begin{gathered}
M_{b}(q, r)=\max \{\mathrm{d}(\mathrm{Sq}, \mathrm{Tr}), \mathrm{d}(\mathrm{fq}, \mathrm{Sq}), \mathrm{d}(\mathrm{gr}, \mathrm{Tr}), \\
\left.\frac{\mathrm{d}(\mathrm{gr}, \mathrm{Tr})+\mathrm{d}(\mathrm{fq}, \mathrm{Sq})}{2 b}, \frac{\mathrm{d}(\mathrm{Sq}, \mathrm{gr})+\mathrm{d}(\mathrm{fq}, \mathrm{Tr})}{2 b}\right\} \\
=\max \left\{\mathrm{d}(\mathrm{fq}, \mathrm{q}), \mathrm{d}(\mathrm{fq}, \mathrm{fq},), \mathrm{d}(\mathrm{q}, \mathrm{q}), \frac{\mathrm{d}(\mathrm{q}, \mathrm{q})+\mathrm{d}(\mathrm{fq}, \mathrm{fq},)}{2 b},\right. \\
\left.\frac{\mathrm{d}(\mathrm{fq}, \mathrm{q})+\mathrm{d}(\mathrm{fq}, \mathrm{q})}{2 b}\right\} \\
=\mathrm{d}(\mathrm{fq}, \mathrm{q}) .
\end{gathered}
$$

From (3.1.6), we have

$$
\int_{0}^{b^{\varepsilon} d(f q, q)} \emptyset(t) d t \leq \int_{0}^{\mathrm{d}(\mathrm{fq}, \mathrm{q})} \emptyset(t) d t
$$

From which it follows that $\mathrm{d}(\mathrm{fq}, \mathrm{q})=0$ (because $b^{\varepsilon}>\mathrm{b}>1$ ). Hence, $\mathrm{fq}=\mathrm{Sq}=\mathrm{q}$.

Similarly, it can be shown $\mathrm{gq}=\mathrm{Tq}=\mathrm{q}$.

To prove uniqueness of fixed point, suppose that $\mathrm{p}$ is an another fixed point off, g, S and T. From (3.1.1),

$$
\begin{gathered}
\int_{0}^{b^{\varepsilon} d(q, q)} \emptyset(t) d t=\int_{0}^{b^{\varepsilon} d(f q, g p)} \emptyset(t) d t \leq \\
\int_{0}^{M_{b}(q, p)} \emptyset(t) d t
\end{gathered}
$$

Where,

$$
\begin{gathered}
M_{b}(q, p)=\max \{\mathrm{d}(\mathrm{Sq}, \mathrm{Tp}), \mathrm{d}(\mathrm{fq}, \mathrm{Sq}), \mathrm{d}(\mathrm{gp}, \mathrm{Tp}), \\
\left.\frac{\mathrm{d}(\mathrm{gp}, \mathrm{Tp})+\mathrm{d}(\mathrm{fq}, \mathrm{Sq})}{2 b}, \frac{\mathrm{d}(\mathrm{Sq}, \mathrm{gp})+\mathrm{d}(\mathrm{fq}, \mathrm{Tp})}{2 b}\right\} \\
=\max \left\{\mathrm{d}(\mathrm{q}, \mathrm{p}), \mathrm{d}(\mathrm{q}, \mathrm{q},), \mathrm{d}(\mathrm{p}, \mathrm{p}), \frac{\mathrm{d}(\mathrm{p}, \mathrm{p})+\mathrm{d}(\mathrm{q}, \mathrm{q},)}{2 b},\right. \\
\left.\frac{\mathrm{d}(\mathrm{q}, \mathrm{p})+\mathrm{d}(\mathrm{q}, \mathrm{p})}{2 b}\right\} \\
=\mathrm{d}(\mathrm{q}, \mathrm{p}), \text { since } \mathrm{b}>1 .
\end{gathered}
$$

From (3.1.7), we have

$$
\int_{0}^{b^{\varepsilon} d(q, p)} \emptyset(t) d t \leq \int_{0}^{\mathrm{d}(\mathrm{q}, \mathrm{p})} \emptyset(t) d t
$$

From which it follows that $\mathrm{d}(\mathrm{q}, \mathrm{p})=0$ (because $b^{\varepsilon}>\mathrm{b}>1$ ). Hence, $q=p$.

Corollary 3.2 Let $(\mathrm{x}, \mathrm{d})$ be $\mathrm{b}$ - metric space with $\mathrm{s}>1$ and $\mathrm{f}$, $\mathrm{T}: \mathrm{X} \rightarrow \mathrm{X}$ be mappings with such that

$$
\begin{gathered}
\int_{0}^{b^{\varepsilon} d(f x, f y)} \emptyset(t) d t \leq \int_{0}^{M_{b}(x, y)} \emptyset(t) d t \quad \forall \mathrm{x}, \mathrm{y} \in \\
\mathrm{X}
\end{gathered}
$$

Where $\varepsilon>1$ is a constant and $\emptyset:[0, \infty) \rightarrow[0, \infty)$ is Lebesque integrable mapping which is summable on each compact subset of $[0, \infty)$, non-negative and such that for $\mathrm{c}>0$, $\int_{0}^{c} \phi(t) d t>0$ and

$$
\begin{gathered}
\text { (3.2.2) } M_{b}(x, y)=\max \{\mathrm{d}(\mathrm{Tx}, \mathrm{Ty}), \mathrm{d}(\mathrm{fx}, \mathrm{Tx}), \mathrm{d}(\mathrm{fy}, \mathrm{Ty}), \\
\left.\frac{\mathrm{d}(\mathrm{fy}, \mathrm{Ty})+\mathrm{d}(\mathrm{fx}, \mathrm{Tx})}{2 b}, \frac{\mathrm{d}(\mathrm{Tx}, \mathrm{fy})+\mathrm{d}(\mathrm{fx}, \mathrm{Ty})}{2 b}\right\}
\end{gathered}
$$

Suppose that one of the pair $(f, T)$ satisfies the b-(E.A.)property and $T(X)$ is b-closed in X. Then the pair $(f, T)$ has 
a point of Coincidence in X, Moreover, if the pair $(f, T)$ is weakly compatible, then $\mathrm{f}$ and $\mathrm{T}$ have a unique common fixed point.

Corollary 3.3 Let (x, d) be b- metric space with s > 1 and f, $\mathrm{T}: \mathrm{X} \rightarrow \mathrm{X}$ be mappings with such that

$$
\begin{gathered}
\int_{0}^{b^{2} d(f x, f y)} \emptyset(t) d t \leq \int_{0}^{M_{b}(x, y)} \emptyset(t) d t \quad \forall \mathrm{x}, \mathrm{y} \in \\
\mathrm{X}
\end{gathered}
$$

Where $\varepsilon>1$ is a constant and $\emptyset:[0, \infty) \rightarrow[0, \infty)$ is Lebesque integrable mapping which is summable on each compact subset of $[0, \infty)$, non-negative and such that for $\mathrm{c}>0$, $\int_{0}^{c} \emptyset(t) d t>0$ and

$$
\begin{gathered}
M_{b}(x, y)=\max \{\mathrm{d}(\mathrm{Tx}, \mathrm{Ty}), \mathrm{d}(\mathrm{fx}, \mathrm{Tx}), \mathrm{d}(\mathrm{fy}, \mathrm{Ty}), \\
\left.\frac{\mathrm{d}(\mathrm{fy}, \mathrm{Ty})+\mathrm{d}(\mathrm{fx}, \mathrm{Tx})}{2 b}, \frac{\mathrm{d}(\mathrm{Tx}, \mathrm{fy})+\mathrm{d}(\mathrm{fx}, \mathrm{Ty})}{2 b}\right\}
\end{gathered}
$$

Suppose that one of the pair $(f, T)$ satisfies the b-(E.A.)property and $T(X)$ is b-closed in X. Then the pair $(f, T)$ has a point of Coincidence in $X$, Moreover, if the pair $(f, T)$ is weakly compatible, then $\mathrm{f}$ and $\mathrm{T}$ have a unique common fixed point.

Example 3.4 Let $X=[0,2]$ and define $d: X \times X \rightarrow[0, \infty)$ as follows

$$
\mathrm{d}(\mathrm{x}, \mathrm{y})= \begin{cases}0, & x=y \\ (x+y)^{2}, & x \neq y\end{cases}
$$

Then $(X, d)$ is a $b$ - metric space with $b=2$.

Let $\mathrm{f}, \mathrm{g}, \mathrm{S}, \mathrm{T}: \mathrm{X} \rightarrow \mathrm{X}$ are defined by

$$
\begin{aligned}
& \mathrm{f}(\mathrm{x})=\frac{2 x}{3}, \mathrm{~g}(\mathrm{x})=0, \mathrm{~S}(\mathrm{x})=\left\{\begin{array}{rr}
x, & x \in[0,1) \\
1, & x=1 \\
\frac{2}{3}, & x \in(1,2]
\end{array} \text { and } \mathrm{T}(\mathrm{x})=\right. \\
& \frac{x}{2},
\end{aligned}
$$

Clearly, $\mathrm{g}(X)$ is closed, $\mathrm{g}(\mathrm{x}) \subseteq \mathrm{S}(X)$ and $\mathrm{f}(\mathrm{x}) \subseteq \mathrm{T}(X)$.

Let $\left\{x_{n}\right\}$ be the sequence in $\mathrm{X}$ such that $x_{n}=1+\frac{1}{n+2}, \mathrm{n}$ $=0,1,2, \ldots$.

So that the pair (f, $S$ ) is non-compatible since $\lim _{n \rightarrow \infty} d\left(f S x_{n}, S f x_{n}\right) \neq 0$.

But satisfies the b-(E.A.)- property since $\lim _{n \rightarrow \infty} f x_{n}=$ $\lim _{n \rightarrow \infty} S x_{n}=\frac{2}{3}$

To check the inequality (3.1.1), for all $\mathrm{x}, \mathrm{y} \in \mathrm{X}$ and $\varepsilon=2$, If $\mathrm{x}=0$, then (3.1.1) is satisfied.

If $\mathrm{x} \in(0,1)$, then

$$
\begin{aligned}
b^{\varepsilon} d(f x, g y)=2^{2} \cdot\left(\frac{2 x}{3}\right)^{2} & =\left(\frac{4 x}{3}\right)^{2} \leq\left(\frac{2 x}{3}+x\right)^{2}=\mathrm{d}(\mathrm{fx}, \mathrm{Sx}) \\
& \leq M_{b}(x, y)
\end{aligned}
$$

If $\mathrm{x}=1$, then

$b^{\varepsilon} d(f x, g y)=2^{2} \cdot\left(\frac{2}{3}\right)^{2}=\frac{16}{9} \leq \frac{25}{9}=\mathrm{d}(\mathrm{fx}, \mathrm{Sx}) \leq M_{b}(x, y)$ If $\mathrm{x} \in(1,2]$, then

$$
\begin{gathered}
b^{\varepsilon} d(f x, g y)=2^{2} \cdot\left(\frac{2 x}{3}\right)^{2}=\left(\frac{4 x}{3}\right)^{2} \leq\left(\frac{2 x}{3}+\frac{2}{3}\right)^{2}=\mathrm{d}(\mathrm{fx}, \mathrm{Sx}) \\
\leq M_{b}(x, y)
\end{gathered}
$$

Thus (3.1.1) is satisfied for all $x, y \in X$. The pairs $(f ; S)$ and $(g ; T)$ are weakly compatible. Hence, all the conditions of Theorem 3.1 are satisfied.
Moreover 0 is the unique common fixed point of $f ; g ; S$ and $T$.

4. In this section we proved well-posedness result in b- metric spaces

Definition 4.1 (Boriceanu et al. 2010) [9] Let (X, d) be a bmetric space and $\mathrm{f}: \mathrm{X} \rightarrow \mathrm{X}$ be a mapping. The fixed point problem is of $\mathrm{f}$ is said to be well-posed iff

(i) $\mathrm{f}$ has a unique fixed point $\mathrm{q}$ in $\mathrm{X}$.

(ii) If $x_{n} \in \mathrm{X}, \quad \mathrm{n} \in \mathrm{N}$ and $\mathrm{d}\left(x_{n}, T x_{n}\right) \rightarrow 0$ as $\mathrm{n} \rightarrow \infty$, then $\mathrm{d}\left(x_{n}, q\right) \rightarrow 0$ as $\mathrm{n} \rightarrow \infty$.

Theorem 4.2 Let (x, d) be b- metric space with $\mathrm{s}>1$ and $\mathrm{f}$, $\mathrm{g}, \mathrm{S}, \mathrm{T}: \mathrm{X} \rightarrow \mathrm{X}$ be mappings. Suppose that all the hypothesis of Theorem 3.1 hold. Then the fixed point problem is well posed for mappings $\mathrm{f}, \mathrm{g}, \mathrm{S}$ and $\mathrm{T}$.

Proof : By Theorem 3.1, the mappings $\mathrm{f}, \mathrm{g}, \mathrm{S}$ and $\mathrm{T}$ have a unique common fixed point, say $\mathrm{q}$ in $\mathrm{X}$. Let $\left\{x_{n}\right\}$ be a sequence in $\mathrm{X}$ such that

$$
\begin{gathered}
\lim _{n \rightarrow \infty} d\left(f x_{n}, x_{n}\right)=\lim _{n \rightarrow \infty} d\left(g x_{n}, x_{n}\right)=\lim _{n \rightarrow \infty} d\left(S x_{n}, x_{n}\right)= \\
\lim _{n \rightarrow \infty} d\left(T x_{n}, x_{n}\right)=0 .
\end{gathered}
$$

Using (3.1.1) and b-triangular inequality, we have

$$
\begin{gathered}
\int_{0}^{\mathrm{d}\left(\mathrm{q}, x_{n}\right)} \emptyset(t) d t=\int_{0}^{\mathrm{d}\left(\mathrm{fq}, x_{n}\right)} \emptyset(t) d t \leq \\
\int_{0}^{s\left[\mathrm{~d}\left(\mathrm{fq}, g x_{n}\right)+\mathrm{d}\left(\mathrm{g} x_{n}, x_{n}\right)\right]} \emptyset(t) d t \\
\text { (4.2.1) } \quad \int_{0}^{\mathrm{d}\left(\mathrm{q}, x_{n}\right)} \emptyset(t) d t \leq \int_{0}^{\frac{s}{s} M_{s}\left(q, x_{n}\right)+\operatorname{sd}\left(\mathrm{g} x_{n}, x_{n}\right)} \emptyset(t) d t
\end{gathered}
$$

Where,

$$
\begin{gathered}
M_{b}\left(q, x_{n}\right)=\max \\
\left\{\begin{array}{c}
\mathrm{d}\left(\mathrm{Sq}, \mathrm{T} x_{n}\right), \mathrm{d}(\mathrm{f} q, \mathrm{~S} q), \mathrm{d}\left(\mathrm{g} x_{n}, \mathrm{~T} x_{n}\right), \frac{\mathrm{d}\left(\mathrm{g} x_{n}, \mathrm{~T} x_{n}\right)+\mathrm{d}(\mathrm{f} q, \mathrm{~S} q)}{2 b}, \\
\left.\frac{\mathrm{d}\left(\mathrm{S} q, \mathrm{~g} x_{n}\right)+\mathrm{d}\left(\mathrm{fq}, \mathrm{T} x_{n}\right)}{2 b}\right\} \\
=\max \left\{\mathrm{d}\left(\mathrm{q}, \mathrm{T} x_{n}\right), 0, \mathrm{~d}\left(\mathrm{~g} x_{n}, \mathrm{~T} x_{n}\right), \frac{\mathrm{d}\left(\mathrm{g} x_{n}, \mathrm{~T} x_{n}\right)+0}{2 b},\right. \\
\left.\frac{\mathrm{d}\left(\mathrm{q}, \mathrm{g} x_{n}\right)+\mathrm{d}\left(\mathrm{q}, \mathrm{T} x_{n}\right)}{2 b}\right\} \\
=\max \left\{\mathrm{d}\left(\mathrm{q}, \mathrm{T} x_{n}\right), 0, \mathrm{~b}\left[\mathrm{~d}\left(\mathrm{~g} x_{n}, \mathrm{q}\right)+\right.\right. \\
\left.\left.\mathrm{d}\left(\mathrm{q}, \mathrm{T} x_{n}\right)\right], \frac{\left.\mathrm{b} \mathrm{d}\left(\mathrm{g} x_{n}, \mathrm{q}\right)+\mathrm{d}\left(\mathrm{q}, \mathrm{T} x_{n}\right)\right]}{2 b}, \frac{\mathrm{d}\left(\mathrm{q}, \mathrm{g} x_{n}\right)+\mathrm{d}\left(\mathrm{q}, \mathrm{T} x_{n}\right)}{2 b}\right\} \\
=\mathrm{b}\left[\mathrm{d}\left(\mathrm{g} x_{n}, \mathrm{q}\right)+\mathrm{d}\left(\mathrm{q}, \mathrm{T} x_{n}\right)\right]
\end{array}\right.
\end{gathered}
$$

Thus from (4.2.1), we have

$$
\begin{gathered}
(4.2 .2) \quad \int_{0}^{\mathrm{d}\left(\mathrm{q}, x_{n}\right)} \emptyset(t) d t \leq \\
\int_{0}^{\frac{b^{2}}{b^{\varepsilon}}\left[\mathrm{d}\left(\mathrm{g} x_{n}, \mathrm{q}\right)+\mathrm{d}\left(\mathrm{q}, \mathrm{T} x_{n}\right)\right]+\operatorname{bd}\left(\mathrm{g} x_{n}, x_{n}\right)} \emptyset(t) d t
\end{gathered}
$$

Again by b- triangular inequality and (4.2.2), we have

$$
\text { (4.2.3) } \int_{0}^{\mathrm{d}\left(\mathrm{q}, x_{n}\right)} \emptyset(t) d t \leq
$$

$$
\left.\int_{0}^{b^{3}} \frac{b^{3}}{\varepsilon} \mathrm{d}\left(\mathrm{g} x_{n}, x_{n}\right)+2 \mathrm{~d}\left(x_{n}, \mathrm{q}\right)+\mathrm{d}\left(x_{n}, \mathrm{~T} x_{n}\right)\right]+\operatorname{bd}\left(\mathrm{g} x_{n}, x_{n}\right) \emptyset(t) d t
$$

So that,

$$
\begin{gathered}
\int_{0}^{\mathrm{d}\left(\mathrm{q}, x_{n}\right)} \emptyset(t) d t \leq \\
\int_{0}^{\frac{1}{\left(1-\frac{b^{3}}{b^{\varepsilon}}\right)}\left[\left(\frac{b^{3}}{b^{\varepsilon}}+\mathrm{b}\right)\left[\mathrm{d}\left(\mathrm{g} x_{n}, x_{n}\right)+\frac{b^{3}}{b^{\varepsilon}} \mathrm{d}\left(x_{n}, \mathrm{~T} x_{n}\right)\right]\right]} \emptyset(t) d t
\end{gathered}
$$

On taking limit $\mathrm{n} \rightarrow \infty$, we obtain

$$
\lim \int_{0}^{\mathrm{d}\left(\mathbf{q}, x_{n}\right)} \emptyset(t) d t=0
$$

From lemma 2.13, we have $\lim _{n \rightarrow \infty} \mathrm{d}\left(\mathrm{q}, x_{n}\right)=0$

This completes the proof. 


\section{References}

1. Aamri M and Moutawakil D : Some new common fixed point theorems under strict contractive conditions. J Math Anal Appl 270:181-188, (2002).

2. Abbas $M$ and Jungck G: Common fixed point results for non-commuting mappings without continuity in cone metric spaces, J. Math. Anal. Appl. 341, 416420(2008).

3. Aghajani A, Abbas $\mathrm{M}$ and Roshan $\mathbf{J}$ R : Common fixed point of generalized weak contractive mappings in partially ordered $b$ metric spaces. Math Slovaca 4:9419609,(2014).

4. Alghamdi M., Hussain N. and Salimi P. : Fixed point and coupled fixed point theorems on $b$-metric- like spaces J. Inequal. Appl., 402 (2013).

5. Ali M., Kamran T. and Karapnar E : An approach to existence of fixed points of generalized contractive multivalued mappings of integral type via admissible mapping, Abstract and Applied Analysis, vol. 2014, Article ID 141489(2014).

6. Amini and Harandi A: Fixed point theory for quasi-contraction maps in $b$-metric spaces. Fixed Point Theory 15(2):351-358,(2014).

7. Babu GVR, Sailaja P.D.: Common fixed points of $(\psi, \phi)$-weak quasi-contractions with property (E.A). Int J Math Sci Comput 1:29-37, (2011).

8. Bakhtin, I.A. The contraction mapping principle in almost metric spaces. Funct. Anal. 1989, 30, 26-37.

9. Boriceanu M, Bota M, Petruşel A: Multivalued fractals in b-metric spaces. Cent Eur J Math 8(2):367-377, (2010).

10. Bota, M.F., Karapinar, E: A note on some results on multi-valued weakly Jungck mappings in b-metric space. Cent. Eur. J. Math. 11(9), 1711-1712 (2013)

11. Branciari A.: A fixed point theorem for mappings satisfying a general contractive condition of integral type, ijmms, 531536(2002)

12. Czerwik, S. : Contraction mappings in b- metric spaces, Acta Math Inf. Univ. Ostravensis, I, 5-11, (1993).

13. Czerwik, S. Nonlinear set valued contraction mappings in b-metric spaces, Atti Sem. Mat. Fis. Univ. Modena, 46, 263-276, (1998).
14. Ding H.S., Ozturk V, Radenovi S: On some new fixed point results in $b$-rectangular metric spaces. J Nonlinear Sci. Appl 8:378-386, (2015).

15. Edelstein, M : An extension of Banach's contraction principle. Proc. Am. Math. Soc. 12, 7-10 (1961)

16. Hussain N, Shah M.H.; KKM mappings in cone $b$-metric spaces. Comput Math Appl 62:16771684(2011).

17. Jovanovic M, kadelburg $\mathrm{Z}$ and Radenovic S. : Common fixed point results in metric type spaces, Fixed Point Theory Appl, 2010:978121(2010).

18. Jungck G: Compatible mappings and common fixed points. Int J Math Sci 9:771-779, (1986).

19. Khan M.S., Swaleh M, Sessa S: Fixed point theorems by altering distances between the points. Bull Aust Math Soc 30(1),1-9, (1984).

20. Kir M, Kiziltunc H :On some well-known fixed point theorems in $b$-metric spaces. Turk $\mathbf{J}$ Anal Number Theory 1(1), 13-16, (2013).

21. Nazir T, Abbas M : Common fixed points of two pairs of mappings satisfying (E.A)-property in partial metric spaces. J Inequal Appl 2014:237, (2014).

22. Oztirk V. and Radenovic S : Some remarks on b(E.A.)-property in b-metric spaces, Springer Plus 5:544, (2016).

23. Ozturk V, Turkoglu D: Common fixed point theorems for mappings satisfying (E.A)property in $b$-metric spaces. J Nonlinear Sci Appl 8(1),127-1133, (2015).

24. Pant R. P., "Common fixed points of noncommuting mappings", J. Math. Anal. Appl. 188, 436-440 (1994)

25. Rhoades, B.E. : A comparison of various definitions of contractive mappings, Trans. Am. Math. Soc. 226, 257-290 (1977).

26. Shah M., Hussain N. : Nonlinear contractions in partially ordered quasi $b$-metric spaces. Commun. Korean Math. Soc.27(1), 117-128 (2012).

27. Z. Liu, J. Li and S. Kang, Fixed point theorems of contractive mappings of integral type, Fixed Point Theory and Applications, 2013:300(2013).

28. Z. Liu, X. Li, S. Kang and S. Cho, Fixed point theorems for mappings satisfying contractive conditions of integral type and applications, Fixed Point Theory and Applications, 2011:64, (2011). 